\title{
Sturmian expansions for two-electron atomic systems: Singly and doubly excited states
}

\author{
A. L. Frapiccini, ${ }^{1, *}$ J. M. Randazzo, ${ }^{1}$ G. Gasaneo, ${ }^{2}$ and F. D. Colavecchia ${ }^{1}$ \\ ${ }^{1}$ División Colisiones Atómicas, Centro Atómico Bariloche and CONICET, 8400 S. C. de Bariloche, Río Negro, Argentina \\ ${ }^{2}$ Departamento de Física, Universidad Nacional del Sur and CONICET, 8000 Bahía Blanca, Buenos Aires, Argentina
}

(Received 30 April 2009; revised manuscript received 20 May 2010; published 11 October 2010)

\begin{abstract}
We present a configuration interaction (CI) method based on the Sturmian expansion for bound states of a two-electron atomic system. These Sturmian functions are solutions of one-electron quantum mechanical problems, where the eigenvalue is the magnitude of a short-range potential. Also, they fulfill the long-range boundary conditions of Coulomb potentials. We choose to expand the Sturmians of the CI basis using $L^{2}$ Laguerre-type functions. We compute ground and single-excited states energies for $\mathrm{He}$ and $\mathrm{H}^{-}$. Moreover, we are able to obtain energies and widths of double excited states of He, using a Sturmian basis with outgoing boundary conditions. In all cases, our ansatz outperforms other CI calculations, for similar basis size.
\end{abstract}

DOI: $10.1103 /$ PhysRevA.82.042503

PACS number(s): 31.15.ac, 31.10.+z

\section{INTRODUCTION}

One of the most common techniques for $a b$ initio calculations in few-electron systems is the configuration interaction (CI) method [1-3]. It has been used along the years to perform atomic structure calculations $[1,4,5]$ and to analyze collisional processes [5]. The solution of the few-body Schrödinger equation within the $\mathrm{CI}$ approach is expanded in terms of antisymmetric products of one-electron wave functions. Each one of the them is the product of a radial function that accounts for the interparticle interaction and spherical harmonics, which describe the angular dependence for a total angular momentum $L$. The matrix elements of the few-body Hamiltonian are then defined by six-dimensional integrals. Since the angular ones can be computed analytically, the matrix elements reduce to (i) one-dimensional integrals for the kinetic energy and the electron-nucleus interactions and (ii) two-dimensional integrals for the electron-electron repulsion. The CI technique leads to good results for many atomic as well as molecular systems. However, energy and other physically relevant magnitudes computed with CI converge slowly. This is due to a pair of closely related factors: first, electron-electron correlation is not explicitly introduced in the method and, second, the electron-electron Kato cusp conditions are not satisfied [6]. Then, high-precision calculations with CI usually require large basis sets, compared to other approaches that deal with the electronic correlation explicitly [7-14].

Introduction of electronic correlation in the calculation of two-electron bound states can be performed in different ways. For example, one can explicitly include the dependence in the interelectronic coordinate, using relative interparticle coordinates [12,14-17] or perimetric ones [18]. However, this by no means ensures the fulfillment of cusp conditions. Moreover, the extension of these methods to other states that include one or more particles in the continuum is not straightforward (see for example, Ref. [19]).

Even though other strategies have been developed to introduce electron-electron correlation, the traditional CI method

\footnotetext{
*afrapic@cab.cnea.gov.ar
}

is still a method of choice in many situations, for example, ionizing collisions. In fact, most successful techniques to describe collisions are still CI ones, such as the convergent close coupling [20], the $R$ matrix, or the $J$ matrix [5]. In these cases, $\mathrm{CI}$ is a simple way to deal with bound and/or continuum states on equal footing. Furthermore, one can always obtain better results, including relevant physical information in the basis itself. Among all the possible basis sets useful to deal with atomic systems, we select Sturmian functions, which were first used by Shull and Loewdin [1] even when they did not pay attention to their connection with Sturmians problems. Rotenberg used the same functions as Shull and Loewdin $[21,22]$. He gave them the name Sturmian to emphasize their connection with Sturm-Liouville theory. Glöckle made use of them in scattering problems [23]. In 1968, Goscinski [24] presented a rigorous mathematical generalization of the Sturmian functions. He conceived Sturmians as the solutions of a Schrödinger equation where the magnitude of a potential is the eigenvalue, and the energy is a parameter [24-26]. Therefore, the physical wave functions for a given interaction are expanded in these eigenstates. In these works, the theory was established for negative energies (i.e., bound states) and general potentials. However, basis sets for general interactions were not widely developed because few two-body potentials admit closed-form analytical solutions. Instead, most of the applications in the literature until now were restricted to Coulomb Sturmian functions (CSF), with few exceptions such those discussed by G. Rawitscher [27] and J. H. Macek and S. Yu. Ovchinnikov [28,29]. They defined numerical strategies to solve the Sturmian equation for general potentials. We extended these works, introducing a systematic way to generate Sturmians for any physically sound potential with different types of boundary conditions [30-32].

In this contribution we continue our previous work with the application of an optimal Sturmian CI basis for excited states of atomic systems. We have already analyzed the definition of a Sturmian CI basis set with the proper asymptotic form for ground atomic states [32]. Here we further develop the method to accommodate continuum states for the description of some features of the atomic spectrum, such as resonances. Our proposal relies on the fact that the basis functions satisfy a Sturmian equation for the electron-nucleus interaction with 
the right asymptotic condition. Two different potentials are included in the two-particle Sturmian equation. The auxiliary potential introduces the physical aspects of the problem and determines the asymptotic behavior of the functions. The generating potential is a short-range interaction whose magnitude is the eigenvalue of the problem. We have used a finite-difference scheme to transform the Sturmian equation into a matrix generalized eigenvalue problem [32]. This method exhibited excellent results for ground states of two-electron atoms. Moreover, we have also shown recently that the introduction of electron-nucleus cusp conditions by a suitable choice of the auxiliary potential can speed up the convergence of the energies of two-electron ground states [33]. However, this approach requires a careful balance between the range of the generating potential and the size of the numerical grid. Here, we chose to write the solution of the Sturmian equation in terms of products of exponential functions times generalized Laguerre polynomials. Our aim is to demonstrate that this spectral representation overcomes the difficulties of the our previous numerical scheme [32] and allows us to compute the solution of the Sturmian equation for arbitrary large values of the coordinates.

We study the efficiency of this method in the calculation of $\mathrm{He}$ atom and $\mathrm{H}^{-}$ion spectra compared to other calculations. Ground states are computed with negative-energy Sturmians for each electron. However, we focus our attention on excited states. In the case of highly asymmetrical excited states (HAES), one electron is in a much higher excited state than the other one. Therefore, the correlation and cusp condition are less relevant, and better results from the CI method are expected. We also are able to compute autoionizing states of He using outgoing boundary conditions. We obtain excellent results in all these cases. We show that this spectral representation is as efficient as the full numerical one presented before [32]. Moreover, our method is better than the direct use of Laguerre functions as basis sets for the three-body systems [5].

The outline of this work is as follows. In Sec. II we review the Sturm-Liouville theory for two-body states. For atomic system we discuss the boundary conditions to be imposed, and general properties of the Sturmian functions. Then, we introduce the numerical method to be used to obtain the Sturmian basis set by means of a finite, $L^{2}$ expansion in Laguerre functions. In Sec. III we present the CI expansion for the two-electron atomic systems and briefly describe the matrix system and analyze numerical features of the problem. In Sec. IV we present the results in $\mathrm{He}$ and $\mathrm{H}^{-}$systems and their isoelectronic series, including convergence tests on the basis parameters. Finally we draw some conclusions in Sec. V. Atomic units are used unless otherwise stated, i.e., $\hbar=e=m_{e}=1$, and energies are measured in Hartrees.

\section{ONE-ELECTRON STURMIAN BASIS SET}

\section{A. Review of the Sturm-Liouville theory}

The time-independent Schrödinger equation for an electron in the center of mass of a heavy particle, interacting through a potential $V(r)=V_{0}(r)+\beta \bar{V}(r)$ is

$$
\left[-\frac{1}{2} \nabla^{2}+V_{0}(r)-E\right] \Phi(\mathbf{r})=-\beta \bar{V}(r) \Phi(\mathbf{r})
$$

Here the energy $E$ of the system is considered a fixed parameter and $\beta$ is the eigenvalue. We have split the potential $V(r)$ in two terms: $V_{0}$ that might contain a long-range (Coulomb) interaction and $\bar{V}$ that is a central, physically sound short-range potential. We write $\Phi(\mathbf{r})$ in spherical coordinates

$$
\Phi(\mathbf{r})=\frac{1}{r} S_{l}(r) Y_{l m}(\theta, \phi)
$$

using the spherical harmonics $Y_{l m}$ [34]. Then, the Schrödinger equation for the radial part $S_{l}(r)$ is

$$
\left[H_{0}-E\right] S_{l}(r)=-\beta_{l} \bar{V}(r) S_{l}(r),
$$

where the radial Hamiltonian is defined as

$$
H_{0}=-\frac{1}{2} \frac{d^{2}}{d r^{2}}+\frac{l(l+1)}{2 r^{2}}+V_{0}(r) .
$$

Two-point boundary conditions must be set to define the problem completely over the domain of the coordinate $r$. We consider Sturmian functions that are regular at the origin:

$$
S_{l}(r=0)=0 .
$$

The second boundary condition is defined for $r \rightarrow \infty$. Let us assume that the potential $\bar{V}(r)$ goes to zero faster than $1 / r$, for sufficiently large $r=R_{0}$. Then, the Sturmian function is the asymptotic solution of the homogeneous equation

$$
\left[H_{0}-E\right] S_{l}(r)=0 .
$$

We consider also that $V_{0}$ includes at least a Coulomb tail of charge $|Z|$. For negative energy, the asymptotic solution behaves as

$$
S_{l}(r) \stackrel{r \rightarrow \infty}{\longrightarrow} \exp -k r-\frac{Z}{k} \ln (2 k r),
$$

with $k=\sqrt{-2 E}$. On the other hand, for positive energy, standing, outgoing, or incoming wave behavior are possible:

$$
S_{l}(r) \stackrel{r \rightarrow \infty}{\longrightarrow} \begin{cases}F_{l}(r) & \text { standing wave } \\ H_{l}^{ \pm}(r) & (+) \text { outgoing or } \\ & (-) \text { incoming wave }\end{cases}
$$

where $F_{l}$ and $H_{l}$ are the regular and irregular Coulomb wave functions, respectively [35].

Since the short-range potential is assumed to vanish for $r>$ $R_{0}$, Eq. (3) is solved in the domain $r \in\left[0, R_{0}\right]$. This solution is matched to the desired asymptotic behavior at $r=R_{0}$ to obtain the eigenvalues $\beta_{l}=\beta_{n, l}$ for $n=0,1, \ldots, \infty$. The negative energy or standing-wave positive energy requires the condition

$$
S_{l}\left(R_{0}\right)=0 .
$$

For outgoing or incoming boundary conditions, both the solution and its derivative (i.e., the logarithmic derivative) should be continuous at $r=R_{0}$

$$
\left.\frac{1}{S_{l}\left(R_{0}\right)} \frac{d S_{l}(r)}{d r}\right|_{r=R_{0}}=\left.\frac{1}{H_{l}^{ \pm}\left(R_{0}\right)} \frac{d H_{l}^{ \pm}(r)}{d r}\right|_{r=R_{0}} .
$$

The solution of Eq. (3) with boundary conditions at $r=$ 0 and $r=R_{0}$ leads to a finite set of $N$ eigenvalues $\beta_{n, l}$ and eigenfunctions $S_{n, l}(r)$ with $n=0,1, \ldots, N-1$. These eigenvalues and eigenvectors are real valued for negative energy or positive energy with a standing-wave boundary 
condition. However, they become complex for positive energy, with incoming or outgoing boundary conditions.

For all those cases, the Sturm-Liouville theory establishes that closure

$$
\sum_{n} S_{n, l}\left(r^{\prime}\right) \bar{V}(r) S_{n, l}(r)=\delta\left(r^{\prime}-r\right),
$$

and the orthogonality condition

$$
\left\langle S_{n^{\prime}, l}|\bar{V}(r)| S_{n, l}\right\rangle=\int_{0}^{R_{0}} d r S_{n^{\prime}, l}(r) \bar{V}(r) S_{n, l}(r)=\delta_{n^{\prime}, n},
$$

is fulfilled by the eigenfunctions.

We stress the fact that the energy $E$ is an external parameter of the Sturm-Liouville equation. In our method, the short-range potential $\bar{V}$ is defined externally according to the problem under scrutiny. We will further discuss this feature in the next sections.

\section{B. Numerical evaluation of the Sturmian functions}

There are a variety of schemes to obtain the Sturmian functions defined in the precedent section. Recently, we have presented a full numerical method to solve this problem, which relies on the discretization in a uniform grid of the wave functions $S_{n, l}$ [32]. Here we propose a different approach to solve Eq. (3), making use of a $L^{2}$ Laguerre-type basis set $[30,31,36,37]$

$$
\varphi_{j, l}(\lambda, r)=e^{-\lambda r}(2 \lambda r)^{l+1} L_{j}^{2 l+1}(2 \lambda r),
$$

where $L_{j}^{\alpha}$ is a generalized Laguerre polynomial [34]. We also include a free parameter $\lambda$ that can be used to scale the radial coordinate for different states. We expand each Sturmian function in the subspace spanned by a set of $N$ Laguerre-type functions $\left\{\varphi_{j, l}\right\}_{j=0}^{N-1}$ as

$$
S_{n, l}^{N}(r)=\sum_{j=0}^{N-1} a_{j, l}^{n} \varphi_{j, l}(\lambda, r) .
$$

The use of this expansion transforms Eq. (3) into

$$
\mathbf{J}_{l} \mathbf{a}_{n, l}=-\beta_{n, l} \overline{\mathbf{V}}_{l} \mathbf{a}_{n, l},
$$

after projecting onto the basis set. The vector $\mathbf{a}_{n, l}=$ $\left\{a_{0, l}^{n}, a_{1, l}^{n}, \ldots, a_{N-1, l}^{n}\right\}^{T}$ represents the coefficients of each Sturmian $S_{n, l}^{N}$, and we have defined the matrix elements $\left[\mathbf{J}_{l}\right]_{j^{\prime}, j}=$ $\left\langle\varphi_{j^{\prime}, l}\left|H_{0}-E\right| \varphi_{j, l}\right\rangle$ and $\left[\overline{\mathbf{V}}_{l}\right]_{j^{\prime}, j}=\left\langle\varphi_{j^{\prime}, l}|\bar{V}| \varphi_{j, l}\right\rangle$. This expansion includes the natural conditions for the coefficients $a_{-1, l}^{n}=0$ and $a_{N, l}^{n}=0$, as a transformation into the Laguerre space of the boundary radial conditions Eq. (5) and Eq. (9). Diagonalization of (15) gives a set of real eigenvalues $\beta_{n, l}$ with $n=0,1, \ldots, N-1$.

Other asymptotic conditions for positive energy, such as outgoing or incoming waves, can be defined. To this end, it is necessary to replace the boundary condition $a_{N, l}^{n}=0$ by

$$
h_{N, l}^{ \pm} a_{N-1}^{n, l}-h_{N-1, l}^{ \pm} a_{N}^{n, l}=0,
$$

where $h_{n, l}^{ \pm}$are the coefficient expansion of the regularized Coulomb wave functions $H_{l}^{ \pm}$in the Laguerre basis set $[38,39]$. Equation (16) can be understood as the transformation of the logarithmic derivative condition given by Eq. (10) into the Laguerre basis. If the elements of the potential are such that $\left[\overrightarrow{\mathbf{V}}_{l}\right]_{j^{\prime}, j}=0$ for $j^{\prime}, j \geqslant N$, condition (16) is equivalent to solve a generalized eigenvalue problem of the form (15) with $\left[\mathbf{H}_{l}\right]_{j^{\prime}, j}=\left[\mathbf{J}_{l}\right]_{j^{\prime}, j}$ for $j^{\prime}, j \neq N-1$ and

$\left[\mathbf{H}_{l}\right]_{N-1, N-1}=\left[\mathbf{J}_{l}\right]_{N-1, N-1}+\left[\mathbf{J}_{l}\right]_{N-1, N}\left(h_{N, l}^{ \pm} / h_{N-1, l}^{ \pm}\right)$.

A set of complex eigenvalues $\beta_{n, l}(n=0,1, \ldots, N-1)$ is the solution of the matrix system (15).

The orthogonality and closure relations [Eq. (11) and Eq. (12), respectively] within this subspace are

$$
\begin{gathered}
\left\langle S_{n^{\prime}, l}^{N}|\bar{V}| S_{n, l}^{N}\right\rangle=\delta_{n, n^{\prime}} \\
\sum_{n=0}^{N-1} S_{n, l}^{N}\left(r^{\prime}\right) \bar{V}(r) S_{n, l}^{N}(r)=\delta^{N}\left(r-r^{\prime}\right),
\end{gathered}
$$

where $\delta^{N}$ is the representation of the Dirac delta function within this subspace spanned by the $\varphi_{j, l}$. This representation has as a limit the Dirac delta as $N \rightarrow \infty[30,32]$.

\section{BOUND-STATE FUNCTION FOR A TWO-ELECTRON ATOMIC SYSTEM}

Let us now consider the Hamiltonian of two electrons in the Coulomb field of a heavy nucleus of charge $Z$ :

$$
H=-\frac{1}{2} \nabla_{\mathbf{r}_{1}}^{2}-\frac{1}{2} \nabla_{\mathbf{r}_{2}}^{2}-\frac{Z}{r_{1}}-\frac{Z}{r_{2}}+\frac{1}{r_{12}},
$$

where $\left(\mathbf{r}_{1}, \mathbf{r}_{2}\right)$ denote the usual set of electronic coordinates with the radial components $r_{i}=\left|\mathbf{r}_{i}\right|(i=1,2)$, while $r_{12}=$ $\left|\mathbf{r}_{1}-\mathbf{r}_{2}\right|$ is the interelectronic distance. The Schrödinger equation for the wave function $\Psi$ is

$$
H \Psi\left(\mathbf{r}_{1}, \mathbf{r}_{2}\right)=E \Psi\left(\mathbf{r}_{1}, \mathbf{r}_{2}\right),
$$

where $E$ is the total energy of the atomic system.

We can now define a CI expansion using one-electron Sturmians. The wave function for a bound state with a given total angular momentum $L$ and projection $M$ is written as:

$$
\begin{aligned}
\Psi\left(\mathbf{r}_{1}, \mathbf{r}_{2}\right)= & \sum_{l_{1} l_{2}} \mathcal{Y}_{l_{1} l_{2}}^{L M}\left(\widehat{\mathbf{r}}_{1}, \widehat{\mathbf{r}}_{2}\right) \\
& \times \sum_{n_{1}=0}^{N_{1}-1} \sum_{n_{2}=0}^{N_{2}-1} b_{n_{1} n_{2}}^{l_{1} l_{2} L M} \mathcal{A} \frac{S_{n_{1} l_{1}}\left(r_{1}\right)}{r_{1}} \frac{S_{n_{2} l_{2}}\left(r_{2}\right)}{r_{2}},
\end{aligned}
$$

where $b_{n_{1} n_{2}}^{l_{1} l_{2} L M}$ are the expansion coefficients. We introduced the symmetrization operator $\mathcal{A}=\left(1+\epsilon P_{12}\right) / \sqrt{2}$ that accounts for the exchange of the electrons according to the Pauli exclusion principle. The permutation operator $P_{12}$ exchanges coordinates $r_{1} \leftrightarrow r_{2}$ and $\epsilon=1$ for singlet states or $\epsilon=-1$ for triplet states.

The angular part is expressed in terms of the bispherical harmonics [14]

$$
\mathcal{Y}_{l_{1} l_{2}}^{L M}\left(\widehat{\mathbf{r}}_{1}, \widehat{\mathbf{r}}_{2}\right)=\sum_{m_{1} m_{2}}\left\langle l_{1} m_{2} l_{2} m_{2} \mid l_{1} l_{2} L M\right\rangle Y_{l_{1} m_{1}}\left(\widehat{\mathbf{r}}_{1}\right) Y_{l_{2} m_{2}}\left(\widehat{\mathbf{r}}_{2}\right)
$$


where $Y_{l m}$ are spherical harmonics and $\left\langle l_{1} m_{2} l_{2} m_{2} \mid l_{1} l_{2} L M\right\rangle$ are Clebsch-Gordan coefficients [14]. The individual angular momentum $l_{1}$ and $l_{2}$ must satisfy the triangular rule and also parity conservation $(-1)^{L}=(-1)^{l_{1}+l_{2}}$. To avoid redundancies in the expansion (22), the angular momenta are restricted to $l_{1} \leqslant l_{2}$. Also, if $l_{1}=l_{2}$, then $n_{1} \leqslant n_{2}$. These restrictions ensure numerical stability in the calculation, avoiding repeated sets of equations given by symmetry.

To solve Eq. (21) by means of the expansion (22), first we observe that for each coordinate, the terms containing the kinetic energy plus the Coulomb interaction are removed from the Hamiltonian (20) if we take $V_{0}=-Z / r$ in the one-dimensional Schrödinger equation (3) for each Sturmian. The remaining term that couples the radial part is the electronelectron repulsion, which can be also expanded in spherical harmonics as

$$
\frac{1}{r_{12}}=\sum_{l=0}^{\infty} \sum_{m=-l}^{l} \frac{4 \pi}{2 l+1} \frac{r_{<}^{l}}{r_{>}^{l+1}} Y_{l m}^{*}\left(\widehat{\mathbf{r}}_{1}\right) Y_{l m}\left(\widehat{\mathbf{r}}_{2}\right),
$$

with $r_{<}=\min \left(r_{1}, r_{2}\right)$ and $r_{>}=\max \left(r_{1}, r_{2}\right)$.

Projecting Eq. (21) onto the basis set, we obtain a generalized matrix system of the form

$$
\mathbf{H b}=E \mathbf{S b},
$$

where $\mathbf{b}$ is a vector containing the expansion coefficients $b_{n_{1} n_{2}}^{l_{1} l_{2} L M}$ for fixed $(L, M), E$ are the energy eigenvalues and the Hamiltonian, and overlapping matrices $\mathbf{H}$ and $\mathbf{S}$ are

$$
\begin{aligned}
& H_{n_{1}^{\prime} n_{2}^{\prime} n_{1} n_{2}}^{l_{1}^{\prime} l_{2} l_{1} l_{2}}=\left[\left(E_{1}+E_{2}\right) O_{n_{1}^{\prime} n_{1}}^{l_{1}^{\prime} l_{1}} O_{n_{2}^{\prime} n_{2}}^{l_{2}^{\prime} l_{2}}-\beta_{n_{1} l_{1}} \bar{V}_{n_{1}^{\prime} n_{1}}^{l_{1}^{\prime} l_{1}} O_{n_{2}^{\prime} n_{2}}^{l_{2}^{\prime} l_{2}}\right. \\
& \left.-\beta_{n_{2} l_{2}} \bar{V}_{n_{2}^{\prime} n_{2}}^{l_{1}^{\prime} l_{2}} O_{n_{1}^{\prime} n_{1}}^{l_{1}^{\prime} l_{1}}\right] \delta_{l_{1}^{\prime} l_{1}} \delta_{l_{2}^{\prime} l_{2}} \\
& +\sum_{l=0}^{\infty} \frac{4 \pi}{2 l+1} R_{n_{1}^{\prime} n_{2}^{\prime} n_{1} n_{2}}^{l_{1}^{\prime} l_{2}^{\prime} l_{1} l_{2} l} \sum_{m=-l}^{l} A_{l_{1}^{\prime} l_{2}^{\prime} l_{1} l_{2} l}^{L M} \\
& +\epsilon P\left(n_{1} \rightarrow n_{2}, l_{1} \rightarrow l_{2}\right) \\
& S_{n_{1}^{\prime} n_{2}^{\prime} n_{1} n_{2}}^{l_{1}^{\prime} l_{2}^{\prime} l_{1} l_{2}}=O_{n_{1}^{\prime} n_{1}}^{l_{1}^{\prime} l_{1}} O_{n_{2}^{\prime} n_{2}}^{l_{2}^{\prime} l_{2}} \delta_{l_{1}^{\prime} l_{1}} \delta_{l_{2}^{\prime} l_{2}}+\epsilon P\left(n_{1} \rightarrow n_{2}, l_{1} \rightarrow l_{2}\right) \text {, }
\end{aligned}
$$

where the matrix elements are defined as

$$
\begin{gathered}
O_{n^{\prime} n}^{l^{\prime \prime} l}=\int_{0}^{\infty} d r S_{n^{\prime} l^{\prime}}(r) S_{n l}(r) \\
V_{n^{\prime} n}^{l^{\prime} l}=\int_{0}^{\infty} d r S_{n^{\prime} l^{\prime}}(r) \bar{V}(r) S_{n l}(r) \\
R_{n_{1}^{\prime} n_{2}^{\prime} n_{1} n_{2}}^{l_{1}^{\prime} l_{2} l_{1} l_{2} l}=\int_{0}^{\infty} d r_{1} \int_{0}^{\infty} d r_{2} S_{n_{1}^{\prime} l_{1}^{\prime}}\left(r_{1}\right) S_{n_{2}^{\prime} l_{2}^{\prime} l_{2}}\left(r_{2}\right) \\
\times \frac{r_{<}^{l}}{r_{>}^{l+1}} S_{n_{1} l_{1}}\left(r_{1}\right) S_{n_{2} l_{2}}\left(r_{2}\right) \\
A_{l_{1}^{\prime} l_{2}^{\prime} l_{1} l_{2} l}^{L M}=\int d \widehat{\mathbf{r}}_{1} \int d \widehat{\mathbf{r}}_{2}\left[\mathcal{Y}_{l_{1}^{\prime} l_{2}^{\prime} l_{2}}^{L M}\left(\widehat{\mathbf{r}}_{1}, \widehat{\mathbf{r}}_{2}\right)\right]^{*} Y_{l m}^{*}\left(\widehat{\mathbf{r}}_{1}\right) \\
\times Y_{l m}\left(\widehat{\mathbf{r}}_{2}\right) \mathcal{Y}_{l_{1} l_{2}}^{L M}\left(\widehat{\mathbf{r}}_{1}, \widehat{\mathbf{r}}_{2}\right)
\end{gathered}
$$

and the operator $P\left(n_{1} \rightarrow n_{2}, l_{1} \rightarrow l_{2}\right)$ exchanges the indexes for symmetry. Note that in Eq. (26), $E_{1}$ and $E_{2}$ are one- electron parameter energies used to compute the basis set, see Eq. (1).

For ground states (a symmetric state with $L=M=0$ ), the total size of the radial two-electron basis is $N_{\text {tot }}=N(N+$ 1)/2 for each pair of electronic angular momenta $\left(l_{1}, l_{2}\right)$. The matrices $\mathbf{H}$ and $\mathbf{S}$ are real and symmetric. While $\mathbf{H}$ is a full matrix due to the interelectronic term, $\mathbf{S}$ has a block diagonal structure corresponding to the overlapping terms for a given pair $\left(l_{1}, l_{2}\right)$. In the HAES case, if $l_{1} \neq l_{2}$ the radial size is $N_{\text {tot }}=$ $N_{1} \times N_{2}$ and if $l_{1}=l_{2}$, then $N_{\text {tot }}=N_{1} \times N_{2}-N_{1}\left(N_{1}-1\right) / 2$. $\mathbf{H}$ and $\mathbf{S}$ will be real and the last one will still have a block diagonal structure for the HAES, but neither of them is going to be symmetric.

So far, we have considered the energy of the basis set as a real, negative parameter, so the Sturmian functions have an exponentially decaying asymptotic behavior. However, if the energy is chosen to be positive, outgoing wave asymptotic behavior can be imposed to the basis set, which is adequate for doubly excited, autoionizing states. The matrix obtained with this boundary condition is now complex symmetric and non-Hermitian, and the eigenvalues will be complex, too.

\section{RESULTS}

\section{A. Sturmian basis-set parameters}

The Sturmian for each electron can be defined for different sets of basis parameters $(\bar{V}, \lambda, E, N)$. Our basis can represent symmetric states where the electrons are equivalent, such as the ground state. It can also describe asymmetric excited states (including HAES), where one electron has a lower energy than the other one. We will use the notation $E_{i}, \lambda_{i}$, and $N_{i}$ for $i=1,2$ to distinguish the electrons. When the electrons are considered on equal footing, we will drop the $i$ subindex and simply write $E_{1}=E_{2}=E$ and so on. However, we will show that different basis parameters might be convenient to deal with continuum (for example, autoionizing) states.

First, we analyze the role of the different basis parameters and its physical relation with the system under scrutiny. One aspect that should be clarified is how the asymptotic behavior of the basis helps to accelerate the convergence of the calculation. The asymptotic behavior of the basis is defined by the energy $E$ and the charge of the long-range potential $V_{0}$, according to Eqs. (6) and (7).

The choice of the energy of the basis will also depend on the state we are dealing with. For example, for the symmetric ground state, we chose $E=E_{\text {approx }} / 2$, where $E_{\text {approx }}$ is an estimate of the energy to be obtained, which can be found in literature according to the atomic system to solve. In the usual case where ones does not know a priori a good approximate for the energy, an iterative method can be implemented. One can start with some arbitrary energy $E_{0}$, obtain the groundstate energy $E_{1}$, recalculate the basis using $E=E_{1}$ and the ground-state energy $E_{2}$, and so on. To set the energy basis of HAES states, we have already pointed out that we consider that one electron is in a lower state than the other. Again if $E_{\text {approx }}$ is an estimate of the energy, and a value $E_{1}$ is selected for the higher state; then simply $E_{2}=E_{\text {approx }}-E_{1}$. For these states, several combinations of $\left(E_{1}, E_{2}\right)$ can be tested until a good energy value for the atomic system is obtained. 
One can select the charge of the Coulomb potential $V_{0}$ as the nuclear charge of the atom and choose the energies $E_{1,2}$ to be the energy of the state considered. In such cases, the basis will have the right asymptotic behavior of all the particles far from each other (in the $\Omega_{0}$ region discussed in Ref. [40]). The election of $V_{0}$ as this Coulomb potential removes the electron-nucleus interactions and provides the correct logarithmic dependence on $r_{1}$ and $r_{2}$ to the wave function in this region. Then, it is clear that a better convergence will result from these CI calculations if we define the basis set such that it describes accurately the inner region of the coordinate space, that is, when two or more particles are close to each other.

The aggregation of the basis in the inner region (that is, the accumulation of probability density of the Sturmians) is controlled by the choice of generator potential $\bar{V}$. For the atomic systems that we are considering, a Yukawa $\bar{V}(r)=$ $-e^{-\alpha r} / r$ potential can be optimal because (i) it does not affect the asymptotic region and (ii) combined with the auxiliary potential $V_{0}$ represents quite well the screening effect produced by one electron over the other. The parameter $\lambda$ defines the range of the Yukawa potential and, hence, determines the aggregation of the basis. It can be seen that in this case the basis-set functions diagonalize Herman-Skillmanlike potentials with many different charges. At this point it is important to note that this is a relevant difference with the standard CSF approach: While our Sturmians are mostly located in the region of the space where the atomic state is non-negligible, CSF are spread in a larger region where the state is already zero.

Finally, we need to choose the scaling parameter $\lambda$ carefully. Here, we performed a study of the ground-state energy for $l_{1}=$ $l_{2}=0$ as a function of $\lambda$ with all other parameters held fixed. In Fig. 1 we see the results for the He atom as a function of $\lambda$. It is clear that for a given set of parameters the energy converges to a minimum. This allows us to define a convergence rule for this parameter in the following calculations, seeking for the value of $\lambda_{1}=\lambda_{2}$ that gives the best ground-state energy eigenvalue. This rule will be extended in the next sections to the asymmetric cases where $\lambda_{1} \neq \lambda_{2}$.

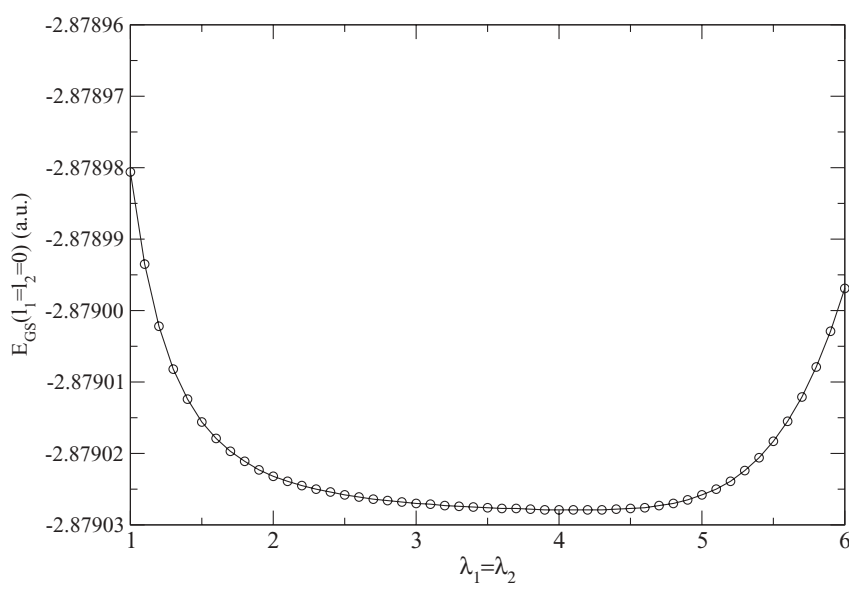

FIG. 1. Ground-state energy (in Hartrees) for He with $N=15$, $E=1.45$ a.u., and $\alpha=0.4$ for the Yukawa, as a function of the parameter $\lambda$ (in arbitrary units).
TABLE I. Ground-state energy $E_{0}$ for $\mathrm{He}$ as a function of the electron pair of angular momenta.

\begin{tabular}{|c|c|c|c|c|}
\hline \multirow[b]{2}{*}{$\left(l_{1}, l_{2}\right)$} & \multirow[b]{2}{*}{ Size } & \multicolumn{2}{|c|}{ Present work } & \multirow[b]{2}{*}{ Ref. [5] $E_{0}$} \\
\hline & & $E_{0}$ & $(\lambda)$ & \\
\hline$(0,0)$ & 120 & -2.879027923 & $(4.2)$ & -2.87902797 \\
\hline$(1,1)$ & 240 & -2.900515058 & $(8.8)$ & -2.90051386 \\
\hline$(2,2)$ & 360 & -2.902772510 & $(13.2)$ & -2.90276209 \\
\hline$(3,3)$ & 480 & -2.903327013 & $(14.4)$ & -2.90331321 \\
\hline$(4,4)$ & 600 & -2.903524584 & $(15.6)$ & -2.90350682 \\
\hline$(5,5)$ & 720 & -2.903613398 & $(18.1)$ & -2.90358925 \\
\hline$(6,6)$ & 840 & -2.903660341 & $(22.2)$ & -2.90362816 \\
\hline \multicolumn{2}{|c|}{ Ref. [7] } & \multicolumn{3}{|c|}{-2.9037243770} \\
\hline
\end{tabular}

\section{B. Ground-state energy of $\mathrm{He}$ and $\mathrm{H}^{-}$}

As usual with CI atomic calculations, we first test our basis computing the ground-state energy of $\mathrm{He}$ and $\mathrm{H}^{-}$. In Table I we summarize the converged energies as several pairs of electron angular momenta are included in the basis. The size in the table stands for the total two-electron basis size. The parameters were set as $\alpha=0.4$ for the short-range Yukawa, $E=-1.45$ a.u. The number of Laguerre functions used for each one-electron basis elements was $N=15$. The size of the basis set was chosen such that, for the first pair of angular momentum, we obtain a value close to that of Ref. [5], where they used a CSF basis with $N=30$ for each electron. As we add more angular momentum pairs, our results improve over those of Ref. [5] in the fourth significant digit. The accuracy of the energies is about $2.2 \times 10^{-5}$ a.u. for this basis size. These results show that our Sturmians enhances the convergence rate of the energy. This boost is largely due to the fact that the basis is denser in the region where the electronic density of the state is more significant. Moreover, it can be attributed also to the proper physics included in the basis, in the sense that it introduces electronic screening and possesses an asymptotic behavior closer to the atomic ground state.

Table II shows the convergence of the energy of the ground state for the $\mathrm{H}^{-}$system. The basis parameters were $\alpha=0.2$, $E=-0.27$ a.u., and $N=20$ for one-electron basis. A bigger basis is needed as the electron-electron correlation is more important for this system. However, the number of elements

TABLE II. Ground-state energy for $\mathrm{H}^{-}$as a function of the electron pair of angular momenta.

\begin{tabular}{lcccc}
\hline \hline & & \multicolumn{2}{c}{ Present work } & \\
\cline { 3 - 4 }$\left(l_{1}, l_{2}\right)$ & Size & $E_{0}$ & $(\lambda)$ & Ref. [5] $E_{0}$ \\
\hline$(0,0)$ & 210 & -0.51449610 & $(1.2)$ & -0.51449614 \\
$(1,1)$ & 420 & -0.52658402 & $(3.7)$ & -0.52658410 \\
$(2,2)$ & 630 & -0.52743848 & $(8.5)$ & -0.52743744 \\
$(3,3)$ & 840 & -0.52762478 & $(9.8)$ & -0.52762391 \\
$(4,4)$ & 1050 & -0.52768776 & $(10.5)$ & -0.52768618 \\
$(5,5)$ & 1260 & -0.52771487 & $(11.3)$ & -0.52771215 \\
$(6,6)$ & 1470 & -0.52772866 & $(14.6)$ & - \\
\multicolumn{5}{c}{ Ref. [7] } \\
\hline \hline
\end{tabular}


TABLE III. Convergence of the $4^{1} F$ and $4^{3} F$ He state energy as a function of the electron pair of angular momenta.

\begin{tabular}{|c|c|c|c|c|c|}
\hline \multirow[b]{2}{*}{$\left(l_{1}, l_{2}\right)$} & \multirow[b]{2}{*}{ Size } & \multicolumn{2}{|c|}{$4^{1} F$} & \multicolumn{2}{|c|}{$4^{3} F$} \\
\hline & & $E_{0}$ & $\left(\lambda_{1}, \lambda_{2}\right)$ & $E_{0}$ & $\left(\lambda_{1}, \lambda_{2}\right)$ \\
\hline$(0,3)$ & 24 & -2.03124998102 & $(2.0,0.4)$ & -2.03125003254 & $(2.0,0.4)$ \\
\hline$(1,2)$ & 48 & -2.03125225658 & $(1.7,0.6)$ & -2.03125229242 & $(2.0,0.7)$ \\
\hline$(1,4)$ & 72 & -2.03125507761 & $(2.0,0.8)$ & -2.03125510143 & $(2.0,0.8)$ \\
\hline$(2,3)$ & 96 & -2.03125509730 & $(1.3,0.4)$ & -2.03125511983 & $(1.2,0.4)$ \\
\hline$(2,5)$ & 120 & -2.03125512692 & $(1.3,0.8)$ & -2.03125514836 & $(1.2,0.7)$ \\
\hline$(3,4)$ & 144 & -2.03125512818 & $(2.0,0.8)$ & -2.03125514924 & $(1.6,0.8)$ \\
\hline$(3,6)$ & 168 & -2.03125512987 & $(2.0,2.0)$ & -2.03125515065 & $(1.6,1.0)$ \\
\hline \multicolumn{2}{|c|}{ Ref. [7] } & \multicolumn{2}{|c|}{-2.03125514438175} & \multicolumn{2}{|c|}{-2.03125516840324} \\
\hline
\end{tabular}

used in our calculations to achieve similar accuracy compared to Ref. [5] is still smaller. The accuracy of the energies is of $4.2 \times 10^{-5}$ a.u. for this basis size.

\section{Singly excited states of $\mathrm{He}$}

Let us now focus onto the main purpose of this contribution: We extend our study to calculate energies of asymmetrical (excited) states. Table III shows the energies of the singlet and triplet states of He for $L=3$, computed with different pairs of angular momenta $\left(l_{1}, l_{2}\right)$. There is an improvement in accuracy and convergence compared to those obtained by Foumouo [5]. The size of the Sturmian basis for each electron is much smaller compared to that of the ground state, and few angular-momenta configurations are needed to achieve convergence. The basis parameters differ for each electron with $E_{1}=-1.95$ a.u., $E_{2}=-0.1$ a.u., $\alpha_{1}=0.1$, and $\alpha_{2}=0.3$ and the one-electron basis sizes are $N_{1}=2$ and $N_{2}=12$. The same parameters were used for singlet and triplet cases. The values of $\left(\lambda_{1}, \lambda_{2}\right)$ for which the energy converges are also shown in Table III. Note that, despite that the energy eigenvalue are similar for the symmetric and antisymmetric states, the parameters are not the same.

The solution of the matrix system given by Eq. (25) generates $N_{\text {tot }}$ energy eigenvalues. Each one of them can be associated to a bound or a continuum state. Since the Sturmian functions form a $L^{2}$ basis set, those states corresponding to continuum ones are pseudostates which ultimately will decay exponentially as the bound states. For the He atom, for example, the eigenvalues which verify $E_{j} \leqslant-2$ will correspond to ground or singly excited states.

We show the results for singly excited states of $\mathrm{He}$ in Table IV, with different values of angular momentum $L$ and levels $n$. The energy values obtained were optimized first for the lowest $n$ level. Although the basis parameters are those which give the best lower energy, they provide a good representation of the excited energy states as well. Of course, this can be improved by changing the basis set values of the free parameter $\lambda$, the energy or the range of the Yukawa potential. However, we want to emphasize that the discretization provided by the finite spectral representation gives a good approximation for the lowest excited states without increasing the size or changing the basis set for each state under scrutiny.
Finally, we point out that the precision of our values increases for higher total angular momenta. As mentioned before, the HAES are expected to give better energies than the ground states for the same number of basis elements, which is confirmed by our calculations.

\section{Doubly excited states for $\mathrm{He}$}

Up to this point, we have shown that our method is able to deal with the ground and HAES for two-electron systems. In these cases, exponential decays are suitable asymptotic behaviors. We show in this section that our method can also be applied to calculate the doubly excited states of a two-electron atom. To this end, we choose a positive energy for the Sturmian basis, and the asymptotic behavior as outgoing wave, to meet the requirements of the doubly excited states where one of the electrons can escape and reach large distances away from the nucleus. In this case both the eigenvalues and the Sturmians will be complex, and the matrix system of Eq. (25) will be complex-symmetric and non-Hermitian. The He eigenvalues $E_{i}$ obtained with the diagonalization will be then complex: the real part is the energies of each atomic state, while the imaginary ones give the width of the state lines. The choice of the basis parameters is made in a similar fashion as in the ground state, but the basis size must be increased for good accuracy.

TABLE IV. Energy of the first three excited states for singlet states of He for different $L$ with a total of 168 basis functions.

\begin{tabular}{cccc}
\hline \hline$n$ & $L$ & Present work & Ref. [7] \\
\hline 3 & 2 & -2.0556110426 & -2.0556207328522456 \\
4 & 2 & -2.0312703879 & -2.0312798461786870 \\
5 & 2 & -2.0197976563 & -2.0200158361599840 \\
4 & 3 & -2.0312551298 & -2.0312551443817490 \\
5 & 3 & -2.0200023656 & -2.0200029371587427 \\
6 & 3 & -2.0137084544 & -2.0138906838155497 \\
5 & 4 & -2.0200007096 & -2.0200007108985847 \\
6 & 4 & -2.0138893011 & -2.0138893453873132 \\
7 & 4 & -2.0101671391 & -2.0102043862247726 \\
6 & 5 & -2.0138890317 & -2.0138890347542797 \\
7 & 5 & -2.0101989535 & -2.0102041828064820 \\
8 & 5 & -2.0075259826 & -2.0078125718286558 \\
\hline \hline
\end{tabular}


TABLE V. Energies for the Rydberg series of the singlet $S$ states of He, using 30 Sturmians per electron.

\begin{tabular}{lccrr}
\hline \hline & \multicolumn{2}{c}{ Present work } & & Ref. [41] \\
\cline { 2 - 4 }$(N, k)_{n}$ & $\Re(\mathrm{E})$ & $\Im(\mathrm{E})$ & & $\Im(\mathrm{E})$ \\
\hline$(2,1)_{2}$ & -0.777876955 & -0.002060106 & -0.777867636 & -0.002270653 \\
$(2,-1)_{2}$ & -0.621817695 & -0.000106535 & -0.621927254 & -0.000107818 \\
$(3,2)_{3}$ & -0.351827523 & -0.001406250 & -0.353538536 & -0.001504906 \\
\hline \hline
\end{tabular}

We emphasize that to apply our method for these boundary conditions, one only needs to change one matrix element in the Hamiltonian, from standing wave conditions $\left(a_{N, l}^{n}=0\right)$ to outgoing ones (17).

In Table $\mathrm{V}$, we present the results for the real and imaginary part of the energy for some of the singlet $S$ states of He. We use the $(N, k)_{n}$ nomenclature for the Rydberg series: the index $N$ denotes the principal quantum number of the remaining ion once the outer electron is ionized. The index $n$ denotes the principal quantum number of the outer electron while the index $k$ determines the parabolic quantum number of the Stark-type state in which the inner electron resides.

The basis set parameters were chosen to give the best value of the first doubly excited state, but it is seen that good results for other states can be achieved. The size of the basis set can be increased not only to obtain more accurate results but also to find more eigenvalues that correspond to the Rydberg series.

\section{E. He isoelectronic series}

Finally, we can go further in the analysis of the basis behavior and study the results for ground and excited states of two-electron atomic systems for different nuclear charges. It is well known that the $\mathrm{H}^{-}$ion has only a double bound (the ground state) and an infinite number of resonant states [42]. On the other hand, $\mathrm{He}$ atom has, in addition to the ground state, an infinite number of single and doubly excited bound states.

The basis set parameters were optimized for each nuclear charge to give the best energy. We used a fixed number of basis elements in every case $\left(N_{1}=N_{2}=20\right)$ to simplify our calculations. In Table VI we show the results for several singly excited energy states as a function of the nuclear charge. Application of our method with double bound asymptotic behavior gives strictly real eigenvalues. However, the calculation with outgoing electrons leads to complex ones. The real part of this eigenvalues is seen in Table VI compared with the results of other authors [43]. In spite of the outgoing wave asymptotic condition, the real part of the complex eigenvalues gives a good result for the ground and singly excited states.

In Table VII we show the results for the doubly excited states $(2,1)_{2}$ and $(2,1)_{3}$, using the notation as in Sec. IV D, for several charges of the isoelectronic series. The results are compared with those of Ref. [44]. We obtained very good agreement in both the real and imaginary parts, with fewer basis elements per electron.

TABLE VI. Real part of the energies for the singlet ground state and first singly excited states of the He-isoelectronic series, using 20 Sturmians per electron.

\begin{tabular}{|c|c|c|c|c|c|c|c|c|}
\hline \multirow[b]{2}{*}{$Z$} & \multicolumn{2}{|c|}{$1 s^{2}$} & \multicolumn{2}{|c|}{$1 s 2 s$} & \multicolumn{2}{|c|}{$1 s 3 s$} & \multicolumn{2}{|c|}{$1 s 4 s$} \\
\hline & Present work & Ref. [43] & Present work & Ref. [43] & Present work & Ref. [43] & Present work & Ref. [43] \\
\hline 2 & -2.9036 & -2.8911 & -2.1459 & -2.1429 & -2.0591 & -2.0603 & -2.0281 & -2.0331 \\
\hline 3 & -7.2798 & -7.2656 & -5.0408 & -5.0331 & -4.7337 & -4.7297 & -4.6287 & -4.6275 \\
\hline 4 & -13.655 & -13.640 & -9.1848 & -9.1735 & -8.5173 & -8.5100 & -8.2868 & -8.2837 \\
\hline 5 & -22.030 & -22.015 & -14.578 & -14.564 & -13.411 & -13.402 & -13.009 & -13.002 \\
\hline 6 & -32.407 & -32.390 & -21.222 & -21.206 & -19.417 & -19.406 & -18.793 & -18.784 \\
\hline
\end{tabular}

TABLE VII. Energies for $(2,1)_{2}$ and $(2,1)_{3}$ doubly excited states of the He isoelectronic series, using 20 Sturmians per electron.

\begin{tabular}{|c|c|c|c|c|c|c|c|c|}
\hline \multirow[b]{3}{*}{$Z$} & \multicolumn{4}{|c|}{$(2,1)_{2}$} & \multicolumn{4}{|c|}{$(2,1)_{3}$} \\
\hline & \multicolumn{2}{|c|}{ Present work } & \multicolumn{2}{|c|}{ Ref. [44] } & \multicolumn{2}{|c|}{ Present work } & \multicolumn{2}{|c|}{ Ref. [44] } \\
\hline & $\Re(E)$ & $\Im(E)$ & $\Re(E)$ & $\mathfrak{s}(\mathrm{E})$ & $\Re(E)$ & $\Im(\mathrm{E})$ & $\mathfrak{R}(\mathrm{E})$ & $\mathfrak{I}(\mathrm{E})$ \\
\hline 2 & -0.77787 & -0.00206 & -0.77787 & -0.00227 & -0.58971 & -0.000652 & -0.58992 & -0.000675 \\
\hline 3 & -1.90661 & -0.00285 & -1.90584 & -0.00283 & -1.41572 & -0.00106 & -1.41557 & -0.00107 \\
\hline 4 & -3.53504 & -0.00301 & -3.53346 & -0.00313 & -2.60258 & -0.00139 & -2.60205 & -0.00132 \\
\hline 5 & -5.65970 & -0.00332 & -5.66088 & -0.00332 & -4.14895 & -0.00142 & -4.14950 & -0.00148 \\
\hline 6 & -8.31744 & -0.00340 & -8.28820 & -0.00345 & -6.05496 & -0.00151 & -6.05800 & -0.00159 \\
\hline
\end{tabular}




\section{CONCLUSIONS}

In this article we presented a CI expansion of two-electron bound states based on one-electron Sturmian functions. These Sturmians are expanded with a finite Laguerre-type basis set. Our proposal allows the introduction of the long-range Coulomb asymptotic behavior both for negative and positive energies. The resulting eigenproblem includes the Coulomb potential and a short-range auxiliary potential which is used to generate the basis. The use of Laguerre-type functions to expand the Sturmians avoids the problems found when using finite difference methods in the diagonalization of the Sturmian equations.

To demonstrate that the use of Laguerre-expanded Sturmian function is as efficient as the other Sturmian methods [32], we study the two-electron atomic system. The accuracy obtained for the ground states of the $\mathrm{He}$ atom and the $\mathrm{H}^{-}$ion are similar to those presented in Ref. [32]. The clue here is that we use the Laguerre functions to solve a one-dimensional problem: the Sturmian equation. Then, the atomic system is solved in terms of these Sturmian functions. This can be understood as a sort of series rearrangement technique which allows the optimization of the basis. In the case of the atomic systems considered, the addition of a short-range Yukawa to the Coulomb potential in the Sturmian basis significantly improves the results over the use of pure Coulomb Sturmians. This makes clear the difference between the use of pure Laguerre basis and Sturmian functions even when they are expanded in terms of Laguerre functions. This is due to the following: (i) the asymptotic behavior of the basis is the proper one to deal with the problem under consideration and (ii) the basis set condenses in the region where the atomic states are mainly located. Moreover, it includes the screening produced by one electron over the other. These facts are of utmost importance when using a CI expansion to build atomic wave functions.

In this article we have also extended our previous work to the calculation of excited states for singlet and triplet states of He for $L=3$ and singly excited states of He, from $L=2$ to $L=5$. This shows the full range of applications of the Sturmian expansion, because different sets of basis parameters can be optimized to obtain either accurate ground or singly excited states.
One of the advantages of our Sturmian method in the study of doubly excited states is the use of basis functions with outgoing wave asymptotic behavior. We obtained very good results for low-lying Rydberg states of singlet He, and more states are expected to converge to the exact value as the number of basis set elements is increased. We would like to emphasize that the complex energies for these states arise naturally from the use of our basis with the proper outgoing condition, and there is no need to artificially manipulate the equation to obtain resonance widths.

A qualitative analysis of ground and singly and doubly excited states was performed for the He isoelectronic series, with real (exponential decay) and complex (outgoing wave) Sturmian basis set. The calculations are in very good agreement for the real part of the energy for the ground, singly excited, and double excited states. The imaginary part of the energy, on the other hand, appears only when the basis set contains elements with outgoing wave behavior. In such cases, a very good approximation of the resonance energy is obtained.

We would like to stress that any kind of short-range potential can be used in our scheme. Our method then can be easily used to analyze isolated or confined atomic systems, as well as artificial (for example, quantum dots) atoms. The choice of the auxiliary potential would then be determined from the physical properties of the problem and can be modified to suite each particular system.

In summary, we have shown that this CI method with generalized Sturmian functions and correct asymptotic conditions included in the basis can outperform other schemes, both in the calculation of bound states or resonances of two-electron species. This implies that bound and continuum states can be treated on equal footing with the same accuracy in our proposal. Furthermore, extension to more electrons presents no difficulty, apart from the algebraic complexity of the description of angular momenta. Applications to molecular calculations and collision problems of our method are on the way and will be presented elsewhere.

\section{ACKNOWLEDGMENTS}

The authors acknowledge the support of Universidad Nacional del Sur (PGI 24/F038) and the Agencia Nacional de Promoción Científica y Técnica PICT 20548/04 and 08/0934.
[1] H. Shull and P. O. Löwdin, J. Chem. Phys. 30, 617 (1959).

[2] C. C. J. Roothaan and A. W. Weiss, Rev. Mod. Phys. 32, 194 (1960).

[3] A. W. Weiss, Phys. Rev. 122, 1826 (1961).

[4] M. W. J. Bromley and J. Mitroy, Int. J. Quantum Chem. 107, 1150 (2007).

[5] E. Foumouo, G. L. Kamta, G. Edah, and B. Piraux, Phys. Rev. A 74, 063409 (2006).

[6] T. Kato, Commun. Pure Appl. Math. 10, 151 (1957).

[7] G. W. F. Drake, Phys. Rev. Lett. 65, 2769 (1990); G. W. F. Drake and Z.-C. Yan, Phys. Rev. A 46, 2378 (1992).

[8] D. P. Carroll, H. J. Silverstone, and R. M. Metzger, J. Chem. Phys. 71, 4142 (1979); W. Kutzelnigg and J. D. Morgan, ibid. 96, 4484 (1992).
[9] C. F. Bunge, Phys. Scr. 21, 328 (1980).

[10] K. V. Rodriguez and G. Gasaneo, J. Phys. B 38, L259 (2005).

[11] T. Li and R. Shakeshaft, Phys. Rev. A 71, 052505 (2005).

[12] K. V. Rodriguez, G. Gasaneo, and D. M. Mitnik, J. Phys. B 40, 3923 (2007).

[13] G. Gasaneo and L. U. Ancarani, Phys. Rev. A 77, 012705 (2008).

[14] G. W. F. Drake, Springer Handbook of Atomic, Molecular, and Optical Physics (Springer, New York, 2006).

[15] W. Kutzelnigg and W. Klopper, J. Chem. Phys. 94, 1985 (1991).

[16] N. Umezawa and S. Tsuneyuki, J. Chem. Phys. 119, 10015 (2003).

[17] A. M. Frolov and V. H. Smith Jr., Phys. Rev. A 53, 3853 (1996).

[18] A. M. Frolov, J. Phys. A 39, 15421 (2006). 
[19] B. Yang, M. Pont, R. Shakeshaft, E. van Duijn, and B. Piraux, Phys. Rev. A 56, 4946 (1997).

[20] I. Bray, D. V. Fursa, A. S. Kheifets, and A. T. Stelbovics, J. Phys. B: At. Mol. Opt. Phys. 35, R117 (2002).

[21] M. Rotenberg, Ann. Phys. (New York) 19, 262 (1962).

[22] M. Rotenberg, Adv. At. Mol. Phys. 6, 233 (1970).

[23] W. Glöckle, J. Hufner, and H. A. Weidenmuller, Nucl. Phys. A 90, 481 (1967).

[24] O. Goscinski, Preliminary Research Report No. 217, Quantum Chemistry Group, Uppsala University (1968).

[25] O. Goscinski, Adv. Quantum Chem. 41, 51 (2002).

[26] J. Avery, J. Avery, and O. Goscinski, Adv. Quantum Chem. 43, 207 (2003).

[27] G. Rawitscher, Phys. Rev. C 25, 2196 (1982).

[28] J. H. Macek and S. Yu. Ovchinnikov, Phys. Rev. A 54, 544 (1996).

[29] S. Yu. Ovchinnikov and J. H. Macek, Phys. Rev. A 55, 3605 (1997).

[30] A. L. Frapiccini, V. Y. Gonzalez, J. M. Randazzo, F. D. Colavecchia, and G. Gasaneo, Int. J. Quantum. Chem. 107, 832 (2007).
[31] A. L. Frapiccini, J. M. Randazzo, G. Gasaneo, and F. D. Colavecchia, Int. J. Quantum. Chem. 110, 963 (2010).

[32] J. M. Randazzo, A. L. Frapiccini, F. D. Colavecchia, and G. Gasaneo, Phys. Rev. A 79, 022507 (2009).

[33] J. M. Randazzo, L. U. Ancarani, G. Gasaneo, A. L. Frapiccini, and F. D. Colavecchia, Phys. Rev. A 81, 042520 (2010).

[34] M. Abramowitz and I. A. Stegun, Handbook of Mathematical Functions (Dover, New York, 1972).

[35] C. J. Joachain, Quantum Collision Theory (North-Holland, Amsterdam, 1983).

[36] J. T. Broad, Phys. Rev. A 31, 1494 (1985).

[37] T. Winata and A. Kartono, Eur. Phys. J. D 28, 307 (2006).

[38] H. A. Yamani and L. Fishman, J. Math. Phys. 16, 410 (1975).

[39] E. J. Heller and H. A. Yamani, Phys. Rev. A 9, 1201 (1974).

[40] H. Klar, Z. Phys. D 16, 231 (1990).

[41] A. Bürgers, D. Wintgen, and J.-M. Rost, J. Phys. B 28, 3163 (1995).

[42] B. H. Bransden and C. J. Joachain, Physics of Atoms and Molecules, 2nd ed. (Prentice-Hall, London, 2003).

[43] J. Avery and J. Avery, J. Math. Chem. 33, 145 (2003).

[44] Y. K. Ho, Phys. Rev. A 23, 2137 (1981). 